\title{
Biomarkers and the prediction of atrial fibrillation: state of the art
}

This article was published in the following Dove Press journal:

Vascular Health and Risk Management

18 July 2016

Number of times this article has been viewed

\author{
Wesley T O'Neal' \\ Sanjay Venkatesh ${ }^{2}$ \\ Stephen T Broughton ${ }^{2}$ \\ William F Griffin ${ }^{3}$ \\ Elsayed Z Soliman ${ }^{4,5}$ \\ 'Department of Medicine, Division \\ of Cardiology, Emory University \\ School of Medicine, Atlanta, GA, \\ USA; ${ }^{2}$ Department of Internal \\ Medicine, Wake Forest School of \\ Medicine, Winston-Salem, NC, USA; \\ ${ }^{3}$ Department of Internal Medicine, \\ Medical University of South Carolina, \\ Charleston, SC, USA; ${ }^{4}$ Department \\ of Internal Medicine, Division of \\ Cardiology, Wake Forest School of \\ Medicine, Winston-Salem, NC, USA; \\ ${ }^{5}$ Epidemiological Cardiology Research \\ Center, Wake Forest School of \\ Medicine, Winston-Salem, NC, USA
}

Correspondence: Wesley T O'Neal Department of Medicine, Division of Cardiology, Emory University School of Medicine, Atlanta, GA 30322, USA

Email wesley.oneal@emory.edu
Abstract: Atrial fibrillation (AF) is the most common sustained arrhythmia encountered in clinical practice, and it places a substantial burden on the health care system. Despite improvements in our understanding of AF pathophysiology, we have yet to develop targeted preventive therapies. Recently, numerous biological markers have been identified to aid in the prediction of future AF events. Subclinical markers of atrial stress, inflammation, endothelial dysfunction, kidney dysfunction, and atherosclerosis have been linked to AF. The connection between these markers and AF is the identification of subclinical states in which AF propagation is likely to occur, as these conditions are associated with abnormal atrial remodeling and fibrosis. Additionally, several risk scores have been developed to aid in the identification of at-risk patients. The practicing clinician should be aware of these subclinical markers, as several of these markers improve the predictive abilities of current AF risk scores. Knowledge of these subclinical markers also provides clinicians with a better understanding of AF risk factors, and the opportunity to reduce the occurrence of AF by incorporating well-known cardiovascular disease risk factor modification strategies. In this review, we highlight several novel biological markers that have improved our understanding of AF pathophysiology and appraise the utility of these markers to improve our ability to predict future AF events.

Keywords: biological markers, prediction, atrial fibrillation

\section{Introduction}

Atrial fibrillation (AF) is the most common sustained arrhythmia encountered in clinical practice, affecting nearly 3 million Americans. ${ }^{1,2}$ Risk factors include diabetes, hypertension, and coronary heart disease. ${ }^{3,4} \mathrm{AF}$ is associated with the development of several cardiovascular outcomes, including stroke, congestive heart failure, and myocardial infarction..$^{5-11}$ Additionally, the arrhythmia is associated with an increased risk of mortality. ${ }^{12-15}$

AF places a significant burden on the United States health care system, with national incremental costs between $\$ 6$ and $\$ 26$ billion. ${ }^{16}$ These estimates likely will increase in the coming decades and parallel the growth in individuals older than 65 years of age, as the prevalence of AF increases dramatically with age. ${ }^{1,17}$ Therefore, preventive strategies with aims to identify those who are at risk for AF development are of paramount importance for public health officials in order to reduce the current and future burden of this arrhythmia and its well-known complications.

Numerous biological markers, either measured in blood or by noninvasive techniques, have been identified to predict the development of future AF events. 
These markers have enhanced our understanding of AF pathophysiology by identifying several processes that facilitate the initiation and perpetuation of the arrhythmia. These markers also provide important prognostic information, especially when faced with the decision to initiate risk factor modification strategies with aims to reduce AF development.

Although several reviews have been written regarding the usefulness of biomarkers in $\mathrm{AF},{ }^{18,19}$ none have focused on the ability of these markers to improve the prediction of incident AF. In this review, we examine several recent biological markers that have improved our understanding of AF pathophysiology and appraise the clinical utility of these markers to predict future AF events (Figure 1).

\section{Markers of atrial stress}

Enlargement of the left atrium is thought to contribute to the abnormal conductive properties observed in AF. ${ }^{20}$ Therefore, markers that detect elevated atrial filling pressures and early atrial hypertension are excellent indicators of abnormal atrial remodeling in which AF development is likely.

B-type natriuretic peptide (BNP) and the stable N-terminal portion of the prohormone, pro-BNP (NT-proBNP), are peptides synthesized by cardiac myocytes in response to elevated pressure and resultant myocardial stretch. ${ }^{21}$ Although commonly thought of as a marker of volume overload and left ventricular dysfunction, direct increases in atrial pressure and stretch have been shown to induce the synthesis and secretion of BNP. ${ }^{22}$

A report from the Cardiovascular Health Study and Malmö Diet and Cancer Study demonstrated that NT-proBNP was significantly associated with incident AF events after adjustment for common risk factors. ${ }^{23,24}$ Data from the
Multi-Ethnic Study of Atherosclerosis found NT-proBNP to be a robust predictor of incident AF in a diverse cohort of racially/ethnically diverse males and females. ${ }^{25}$ Additionally, BNP was shown to predict incident AF in the Framingham Heart Study and this marker improved the predictive ability of the AF risk score developed in this cohort. ${ }^{26}$ BNP also was found to improve the predictive ability of the Cohorts for Heart and Aging Research in Genomic Epidemiology AF consortium (CHARGE-AF) risk score for $\mathrm{AF}^{27}$

\section{Markers of inflammation}

Inflammation has been implicated in the pathophysiology of $\mathrm{AF}^{28}$ and C-reactive protein (CRP) has been the most widely studied proinflammatory marker regarding AF risk. This acute-phase reactant produced in the liver is thought to promote arrhythmiogenesis through atrial remodeling and increasing atrial ectopy, suggesting a link between inflammation and arrhythmiogenesis. ${ }^{29}$

Data from the Cardiovascular Health Study, a populationbased cohort in Norway, and the Malmö Diet and Cancer Study have demonstrated that higher levels of CRP are associated with an increased risk of $\mathrm{AF}^{24,30,31}$ An examination of participants from the Copenhagen City Heart Study observed an increased risk of $\mathrm{AF}$ with higher levels of CRP. ${ }^{32} \mathrm{CRP}$ also has been shown to predict AF after acute myocardial infarction. ${ }^{33}$ Although CRP has been associated with an increased risk for $\mathrm{AF}$, the addition of this inflammatory marker did not improve the predictive abilities of the Framingham Heart Study or CHARGE-AF risk scores for incident $\mathrm{AF}^{26,27}$ Data from the Malmö Diet and Cancer Study also failed to show improvement in the prediction for future AF events beyond traditional risk factors with CRP. ${ }^{24}$

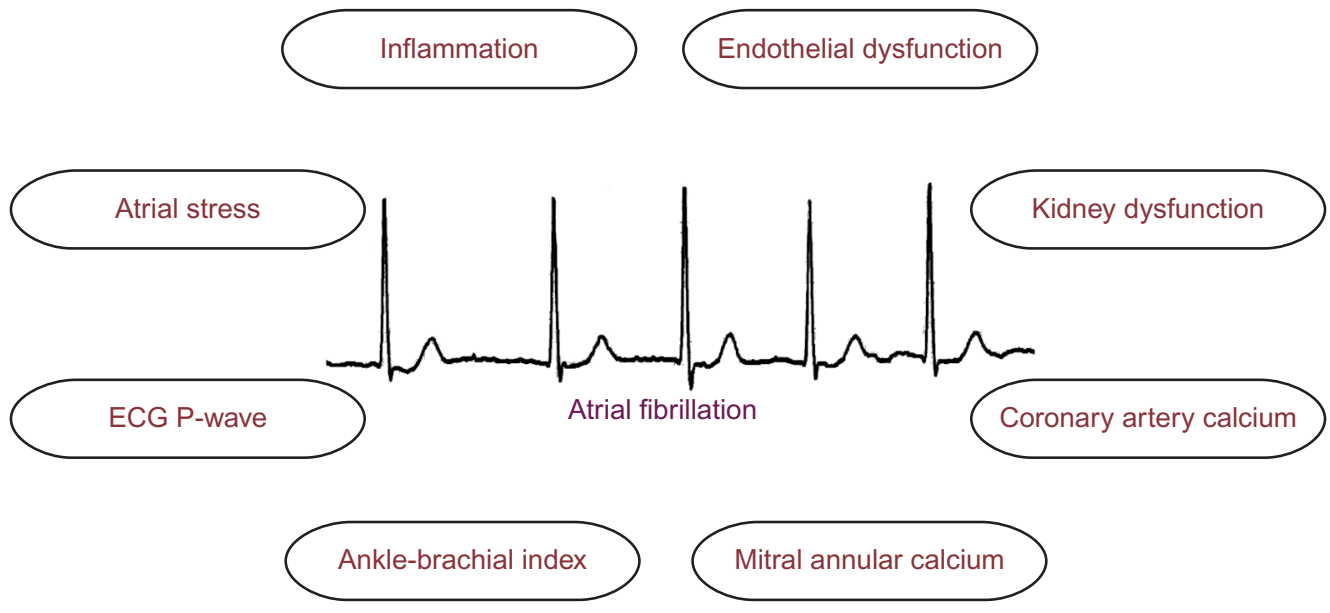

Figure I Biomarkers implicated in the prediction of incident atrial fibrillation. Abbreviation: ECG, electrocardiogram. 


\section{Endothelial dysfunction}

Markers of endothelial dysfunction have been linked to AF. Arterial flow-mediated dilation (FMD) is an indirect measurement of endothelial nitric oxide (NO) release, ${ }^{34}$ and abnormalities in this process have been linked to AF through atrial remodeling and increased atrial ectopy. ${ }^{35,36}$

A study of patients with chronic AF showed that abnormal FMD measurements are significantly impaired compared with sinus rhythm controls. ${ }^{37}$ Another case-control study showed that participants with persistent AF have impaired FMD, and that FMD improves after restoration of sinus rhythm..$^{38}$ Only one report from the Multi-Ethnic Study of Atherosclerosis has demonstrated that abnormal FMD measurements precede the development of AF, suggesting a role for endothelial dysfunction in the pathogenesis of $\mathrm{AF}^{39}$ Although abnormal endothelial dysfunction predisposes to AF, no studies have assessed the ability of this marker to improve the discriminatory capacity of current AF risk scores. Additionally, the use of FMD as an indirect measurement of NO has not been widely adopted as a biological marker for the prediction of arrhythmias.

\section{Markers of kidney dysfunction}

Elevations in serum creatinine and reductions in glomerular filtration rate (GFR) are associated with hypertension, higher levels of inflammation, and cardiovascular disease..$^{40}$ These common comorbid conditions are well-known risk factors for AF development.

Reductions in GFR, as measured by cystatin C, and the presence of albuminuria were associated with an increased risk for incident AF in the Atherosclerosis Risk In Communities study. ${ }^{41}$ Similar results were obtained for GFR measured by serum creatinine, although not as robust as cystatin C..$^{41}$ The association between reductions in GFR by serum creatinine and incident $\mathrm{AF}$ also was observed in a prospective community-based observational cohort study in Japan, ${ }^{42}$ and in a cohort of hypertensive patients. ${ }^{43}$ Markers of inflammation have been shown to predict incident $\mathrm{AF}$ in patients with chronic kidney disease (CKD) ${ }^{44}$ providing evidence that the proinflammatory state of CKD promotes the development of AF. However, no reports have explored the ability of CKD markers to improve the prediction of incident AF events beyond traditional risk factors.

\section{Coronary artery calcium}

Coronary artery calcium (CAC) measured by cardiac computed tomography (CT) provides an estimate of coronary plaque burden. ${ }^{45}$ This technique largely has been used to detect obstructive coronary artery disease, but recent reports have demonstrated that $\mathrm{CAC}$ predicts events that are not limited to the coronary arteries, including stroke. ${ }^{46}$ Given that coronary heart disease is a well-known risk factor for $\mathrm{AF}^{3,4}$ several reports have explored the utility of CAC measurements to predict $\mathrm{AF}$ events.

An examination of 6,641 participants with baseline CAC measurements from the Multi-Ethnic Study of Atherosclerosis has shown that higher levels of coronary calcium predict incident $\mathrm{AF}^{47}$ Additionally, a follow-up study from the same cohort demonstrated that the relationship between CAC and $\mathrm{AF}$ depends on the extent of CAC progression over time. ${ }^{48}$ Highly calcified coronary arteries are associated with larger pulmonary veins and left atrial enlargement, ${ }^{49}$ suggesting that the presence of CAC identifies individuals who have the abnormal substrate for AF propagation. The inclusion of CAC in the Framingham Heart Study and CHARGE AF risk scores for $\mathrm{AF}$ also was shown to improve the predictive abilities of both scores. ${ }^{47}$

\section{Mitral annular calcium}

Mitral annular calcification (MAC) is a chronic degenerative process that affects the base of the mitral valve. Several AF risk factors, such as diabetes and hypertension, have been associated with the presence of MAC. ${ }^{50,51}$ The presence of MAC has been associated with left atrial enlargement, ${ }^{52}$ suggesting that persons with MAC are likely to have the necessary substrate for AF development.

In the Framingham Heart Study, MAC, detected by echocardiography, was associated with the development of $\mathrm{AF}^{53} \mathrm{~A}$ report from the Multi-Ethnic Study of Atherosclerosis also demonstrated that MAC, detected by CT, was associated with an increased risk of $\mathrm{AF}$ and this risk was greater with higher levels of MAC. ${ }^{54}$ Additionally, MAC was shown to improve discrimination beyond variables included in the Framingham Heart Study and CHARGE$\mathrm{AF}$ risk scores for AF.

\section{Ankle-brachial index}

The ankle-brachial index (ABI) has been widely accepted as a diagnostic tool to detect the presence of peripheral artery disease, and abnormalities in this measurement are associated with well-known AF risk factors, such as diabetes and smoking. ${ }^{55,56}$ This tool has been suggested to be a unique biological marker as it has the ability to detect pathology before clinical symptoms are evident, and provides physicians with an opportunity to implement preventive strategies before cardiovascular disease events ensue. ${ }^{57}$ 
Data from the Multi-Ethnic Study of Atherosclerosis have demonstrated that abnormal ABI measurements (ie, $<1.0$ or $>1.4$ ) are associated with an increased risk for AF development. ${ }^{58}$ Similar results were reported from the Cardiovascular Health Study. ${ }^{59}$ Abnormal ABI values and AF are associated with proinflammatory markers and poor cardiovascular risk factor profiles in which each condition likely influences the other. ${ }^{3,30,55}$ Although this measure has not been incorporated into recently developed AF risk scores, abnormalities in the ABI are able to detect persons in whom AF is likely to develop.

\section{Electrocardiographic P-wave}

The P-wave on the resting 12-lead electrocardiogram (ECG) is a representation of atrial electrophysiology. Abnormalities of this marker are thought to represent delayed atrial depolarization due to underlying atrial fibrosis, dilation, and elevated filling pressure. ${ }^{60,61}$ These markers detect abnormal atrial substrate, which allows for AF propagation.

P-wave terminal force in lead $\mathrm{V}_{1}\left(\mathrm{PTFV}_{1}\right)$ is one of the best-known left atrial abnormalities, and this metric is highly correlated with left atrial pressure and size. ${ }^{62,63}$ PTFV $_{1}$ was associated with incident AF in the Atherosclerosis Risk In Communities study. ${ }^{64}$ Additionally, prolonged P-wave duration was predictive of incident AF in the same cohort, ${ }^{64}$ the Framingham Heart Study, ${ }^{65}$ and the Copenhagen ECG Study. ${ }^{66}$ The relationship between P-wave duration and AF was nonlinear, with shortened P-wave duration being predictive of incident AF in the Copenhagen ECG Study. ${ }^{66}$ Similarly, inconsistencies were reported for PR interval, with some studies showing an association between prolonged PR interval and $A F,{ }^{64,67-69}$ and others demonstrating an increased AF risk with short PR interval. ${ }^{69,70}$ Prolonged PR interval was included as a covariate in the Framingham Heart Study risk score for $\mathrm{AF}^{71}$ and $\mathrm{P}$-wave duration was incorporated into the AF risk score developed in the Atherosclerosis Risk In Communities study. ${ }^{72}$ Both reports demonstrate that the ECG is able to improve our ability to predict AF. However, the ability of other $\mathrm{P}$-wave markers to improve AF prediction has not been explored. Nonetheless, these findings implicate the ECG as a cost-effective biological marker of AF risk due to its low-cost and widespread availability.

\section{Current atrial fibrillation risk scores}

To date, three scoring systems have been developed to predict incident AF. These risk scores were developed from community-based studies in which the identification of cardiovascular disease risk factors was the primary focus.
The Framingham Heart Study was one of these cohorts, in which the following clinical characteristics were incorporated into the risk score for AF: age, sex, body mass index, systolic blood pressure, treatment of hypertension, PR interval duration, clinically significant cardiac murmur, and heart failure. ${ }^{71}$ Additionally, a score was developed from the Atherosclerosis Risk In Communities study in which age, race, height, smoking, systolic blood pressure, treatment of hypertension, cardiac murmur, ECG left ventricular hypertrophy, prolonged P-wave duration, diabetes, coronary heart disease, and heart failure were incorporated to develop a 10-year risk score for AF. $^{72}$ The most recent risk score was developed from cohort studies participating in CHARGE-AF. ${ }^{70}$ In this risk score, individual participant data was used from the Framingham Heart Study, the Cardiovascular Health Study, and the Atherosclerosis Risk In Communities study to derive a 5-year predictive model including the following characteristics: age, race, height, weight, systolic and diastolic blood pressure, current smoking, treatment of hypertension, diabetes, and history of myocardial infarction and heart failure. ${ }^{70}$

From these reports, it is evident that a poor cardiovascular risk factor profile portends an increased risk for AF development. However, the risk scores developed from the Framingham Heart Study and the Atherosclerosis Risk In Communities study demonstrate that ECG markers of the left atrium are important predictors of AF. Although PR interval did not improve the CHARGE-AF risk score's ability to predict future AF events, ${ }^{70}$ the aforementioned findings provide evidence that ECG-based markers of atrial pathology are important for AF prediction in certain populations. Additionally, the addition of BNP to the Framingham Heart Study risk score improved the predictive ability of the model. ${ }^{26}$ $\mathrm{CAC}$ and $\mathrm{MAC}$ also were able to improve discrimination in the Framingham Heart Study and CHARGE-AF risk scores for $\mathrm{AF}^{47,54}$ but modest improvements were noted compared with the reclassification statistics reported for BNP. ${ }^{26}$ Overall, these findings support a role for biological markers to improve the prediction of AF events.

\section{Discussion}

In this review, we have identified several biomarkers that predict incident AF. The link between these biological markers and AF is the identification of subclinical states in which AF propagation is likely to occur after an inciting trigger. For example, elevations in BNP detect underlying atrial stretch, which increase one's risk for AF. Similarly, markers of inflammation, endothelial dysfunction, and kidney dysfunction identify those who likely have abnormal atrial remodeling. 
Persons with higher levels of CAC and MAC, and persons with abnormal ABI measurements and $\mathrm{P}$-wave indices also have these abnormal atrial properties. If these processes are not corrected, myocardial fibrosis ensues and results in the abnormal electrophysiological properties in which AF development is probable. ${ }^{73}$ Therefore, each marker explored represents a potential area for preventive intervention.

Due to the current and future burden that AF will place on the health care system, adequate risk assessment is crucial to prevent the development of this common arrhythmia. The aforementioned reports have undoubtedly improved our understanding of AF pathophysiology, and provided clinicians with important information with which to incorporate preventive measures. The practicing clinician should be aware of conditions associated with each of the markers discussed, as they also are associated with an increased risk for cardiovascular disease. An example would be the appropriate treatment of hypertension to reduce cardiovascular disease risk. By appropriately treating this condition, reductions in atrial pressure and atrial remodeling will simultaneously decrease the occurrence of AF. ${ }^{74-76}$ The reduction of subclinical atherosclerosis (ie, coronary calcification) with lipid-lowering therapies is another example to possibly reduce AF risk by decreasing the development of the necessary substrate for arrhythmia propagation. ${ }^{77}$ Also, the identification of abnormal P-wave indices on routine ECG measurement should alert providers to an increased risk for AF development and prompt the implementation of cardiovascular risk factor modification strategies.

From these reports, it is apparent that biomarkers provide important prognostic information regarding AF risk, and potentially have the ability to improve the prediction of this common arrhythmia. Additionally, appropriate risk stratification is needed to identify populations in which targeted preventive therapies will be most beneficial. Further studies are needed to determine if many of the newer biological markers identified in this review are able to refine the predictive abilities of current AF risk scores. Also, the biological markers discussed often represent distinct subclinical states associated with a single comorbid condition. It is possible that multiple biomarkers encompassing several subclinical states will provide a unique profile in which AF risk prediction is possible before the development of clinically apparent disease, and this hypothesis should be explored.

Overall, the identification of biological markers has improved our understanding of AF pathophysiology and demonstrated an ability to improve AF risk prediction. Further research in this area will allow for more precise and personalized strategies to ultimately prevent AF.

\section{Disclosure}

The authors report no conflicts of interest in this work.

\section{References}

1. Go AS, Hylek EM, Phillips KA, et al. Prevalence of diagnosed atrial fibrillation in adults: national implications for rhythm management and stroke prevention: the AnTicoagulation and Risk Factors in Atrial Fibrillation (ATRIA) Study. JAMA. 2001;285(18):2370-2375.

2. Mozaffarian D, Benjamin EJ, Go AS, et al. Executive summary: heart disease and stroke statistics-2016 update: a report from the American heart association. Circulation. 2016;133(4):447-454.

3. Benjamin EJ, Levy D, Vaziri SM, D’Agostino RB, Belanger AJ, Wolf PA. Independent risk factors for atrial fibrillation in a population-based cohort. The Framingham Heart Study. JAMA. 1994;271(11):840-844.

4. Krahn AD, Manfreda J, Tate RB, Mathewson FA, Cuddy TE. The natural history of atrial fibrillation: incidence, risk factors, and prognosis in the Manitoba Follow-Up Study. Am J Med. 1995;98(5):476-484.

5. Wolf PA, Abbott RD, Kannel WB. Atrial fibrillation as an independent risk factor for stroke: the Framingham Study. Stroke. 1991;22(8): 983-988.

6. Gottdiener JS, Arnold AM, Aurigemma GP, et al. Predictors of congestive heart failure in the elderly: the Cardiovascular Health Study. J Am Coll Cardiol. 2000;35(6):1628-1637.

7. Stewart S, Hart CL, Hole DJ, McMurray JJ. A population-based study of the long-term risks associated with atrial fibrillation: 20-year follow-up of the Renfrew/Paisley study. Am J Med. 2002;113(5):359-364.

8. Soliman EZ, Safford MM, Muntner P, et al. Atrial fibrillation and the risk of myocardial infarction. JAMA Intern Med. 2014;174(1):107-114.

9. O'Neal WT, Qureshi W, Zhang ZM, Soliman EZ. Bidirectional association between atrial fibrillation and congestive heart failure in the elderly. J Cardiovasc Med (Hagerstown). 2016;17(3):181-186.

10. Soliman EZ, Lopez F, O’Neal WT, et al. Atrial fibrillation and risk of ST-segment-elevation versus non-ST-segment-elevation myocardial infarction: The Atherosclerosis Risk in Communities (ARIC) study. Circulation. 2015;131(21):1843-1850.

11. O’Neal WT, Sangal K, Zhang ZM, Soliman EZ. Atrial fibrillation and incident myocardial infarction in the elderly. Clin Cardiol. 2014;37(12): 750-755.

12. Benjamin EJ, Wolf PA, D’Agostino RB, Silbershatz H, Kannel WB, Levy D. Impact of atrial fibrillation on the risk of death: the Framingham Heart Study. Circulation. 1998;98(10):946-952.

13. Conen D, Chae CU, Glynn RJ, et al. Risk of death and cardiovascular events in initially healthy women with new-onset atrial fibrillation. JAMA. 2011;305(20):2080-2087.

14. Chamberlain AM, Gersh BJ, Alonso A, et al. Decade-long trends in atrial fibrillation incidence and survival: a community study. Am JMed. 2015;128(3):260-267.

15. O'Neal WT, Efird JT, Judd SE, et al. Impact of awareness and patterns of nonhospitalized atrial fibrillation on the risk of mortality: The reasons for geographic and racial differences in stroke (REGARDS) study. Clin Cardiol. 2016;39(2):103-110.

16. Kim MH, Johnston SS, Chu BC, Dalal MR, Schulman KL. Estimation of total incremental health care costs in patients with atrial fibrillation in the United States. Circ Cardiovasc Qual Outcomes. 2011;4(3):313-320.

17. Odden MC, Coxson PG, Moran A, Lightwood JM, Goldman L, BibbinsDomingo $\mathrm{K}$. The impact of the aging population on coronary heart disease in the United States. Am J Med. 2011;124(9):827-833.

18. Hijazi Z, Oldgren J, Siegbahn A, Granger CB, Wallentin L. Biomarkers in atrial fibrillation: a clinical review. Eur Heart J. 2013;34(20): 1475-1480.

19. Vilchez JA, Roldan V, Hernandez-Romero D, Valdes M, Lip GY, Marin F. Biomarkers in atrial fibrillation: an overview. Int J Clin Pract. 2014;68(4):434-443.

20. Psaty BM, Manolio TA, Kuller LH, et al. Incidence of and risk factors for atrial fibrillation in older adults. Circulation. 1997;96(7):2455-2461.

21. Levin ER, Gardner DG, Samson WK. Natriuretic peptides. $N$ Engl J Med. 1998;339(5):321-328. 
22. Mantymaa P, Vuolteenaho O, Marttila M, Ruskoaho H. Atrial stretch induces rapid increase in brain natriuretic peptide but not in atrial natriuretic peptide gene expression in vitro. Endocrinology. 1993;133(3): $1470-1473$.

23. Patton KK, Ellinor PT, Heckbert SR, et al. N-terminal pro-B-type natriuretic peptide is a major predictor of the development of atrial fibrillation: the Cardiovascular Health Study. Circulation. 2009;120(18): $1768-1774$.

24. Smith JG, Newton-Cheh C, Almgren P, et al. Assessment of conventional cardiovascular risk factors and multiple biomarkers for the prediction of incident heart failure and atrial fibrillation. $\mathrm{J} \mathrm{Am} \mathrm{Coll} \mathrm{Cardiol.}$ 2010;56(21):1712-1719.

25. Patton KK, Heckbert SR, Alonso A, et al. N-terminal pro-B-type natriuretic peptide as a predictor of incident atrial fibrillation in the MultiEthnic Study of Atherosclerosis: the effects of age, sex and ethnicity. Heart. 2013;99(24):1832-1836.

26. Schnabel RB, Larson MG, Yamamoto JF, et al. Relations of biomarkers of distinct pathophysiological pathways and atrial fibrillation incidence in the community. Circulation. 2010;121(2):200-207.

27. Sinner MF, Stepas KA, Moser CB, et al. B-type natriuretic peptide and $\mathrm{C}$-reactive protein in the prediction of atrial fibrillation risk: the CHARGE-AF Consortium of community-based cohort studies. Europace. 2014;16(10):1426-1433.

28. Frustaci A, Chimenti C, Bellocci F, Morgante E, Russo MA, Maseri A. Histological substrate of atrial biopsies in patients with lone atrial fibrillation. Circulation. 1997;96(4):1180-1184.

29. Watanabe T, Takeishi Y, Hirono O, et al. C-reactive protein elevation predicts the occurrence of atrial structural remodeling in patients with paroxysmal atrial fibrillation. Heart Vessels. 2005;20(2):45-49.

30. Aviles RJ, Martin DO, Apperson-Hansen C, et al. Inflammation as a risk factor for atrial fibrillation. Circulation. 2003;108(24):3006-3010.

31. Nyrnes A, Njolstad I, Mathiesen EB, et al. Inflammatory biomarkers as risk factors for future atrial fibrillation. An eleven-year follow-up of 6315 men and women: the tromso study. Gend Med. 2012;9(6):536-547.

32. Marott SC, Nordestgaard BG, Zacho J, et al. Does elevated C-reactive protein increase atrial fibrillation risk? A Mendelian randomization of 47,000 individuals from the general population. $J$ Am Coll Cardiol. 2010;56(10):789-795.

33. Aronson D, Boulos M, Suleiman A, et al. Relation of C-reactive protein and new-onset atrial fibrillation in patients with acute myocardial infarction. Am J Cardiol. 2007;100(5):753-757.

34. Celermajer DS, Sorensen KE, Gooch VM, et al. Non-invasive detection of endothelial dysfunction in children and adults at risk of atherosclerosis. Lancet. 1992;340(8828):1111-1115.

35. Kim YM, Guzik TJ, Zhang YH, et al. A myocardial Nox2 containing NAD(P)H oxidase contributes to oxidative stress in human atrial fibrillation. Circ Res. 2005;97(7):629-636.

36. Lin YK, Lu YY, Chen YC, Chen YJ, Chen SA. Nitroprusside modulates pulmonary vein arrhythmogenic activity. J Biomed Sci. 2010;17(1):20.

37. Freestone B, Chong AY, Nuttall S, Lip GY. Impaired flow mediated dilatation as evidence of endothelial dysfunction in chronic atrial fibrillation: relationship to plasma von Willebrand factor and soluble E-selectin levels. Thromb Res. 2008;122(1):85-90.

38. Skalidis EI, Zacharis EA, Tsetis DK, et al. Endothelial cell function during atrial fibrillation and after restoration of sinus rhythm. Am J Cardiol. 2007;99(9):1258-1262.

39. O'Neal WT, Efird JT, Yeboah J, et al. Brachial flow-mediated dilation and incident atrial fibrillation: the multi-ethnic study of atherosclerosis. Arterioscler Thromb Vasc Biol. 2014;34(12):2717-2720.

40. Sarnak MJ, Levey AS, Schoolwerth AC, et al. Kidney disease as a risk factor for development of cardiovascular disease: a statement from the American Heart Association Councils on Kidney in Cardiovascular Disease, High Blood Pressure Research, Clinical Cardiology, and Epidemiology and Prevention. Circulation. 2003;108(17): 2154-2169.

41. Alonso A, Lopez FL, Matsushita K, et al. Chronic kidney disease is associated with the incidence of atrial fibrillation: the Atherosclerosis Risk in Communities (ARIC) study. Circulation. 2011;123(25):2946-2953.
42. Watanabe H, Watanabe T, Sasaki S, Nagai K, Roden DM, Aizawa Y. Close bidirectional relationship between chronic kidney disease and atrial fibrillation: the Niigata preventive medicine study. Am Heart $J$. 2009;158(4):629-636.

43. Horio T, Iwashima Y, Kamide K, et al. Chronic kidney disease as an independent risk factor for new-onset atrial fibrillation in hypertensive patients. J Hypertens. 2010;28(8):1738-1744.

44. Amdur RL, Mukherjee M, Go A, et al. Interleukin-6 is a risk factor for atrial fibrillation in chronic kidney disease: findings from the CRIC study. PLoS One. 2016;11(2): 0148189.

45. Ghadri JR, Pazhenkottil AP, Nkoulou RN, et al. Very high coronary calcium score unmasks obstructive coronary artery disease in patients with normal SPECT MPI. Heart. 2011;97(12):998-1003.

46. Hermann DM, Gronewold J, Lehmann N, et al. Coronary artery calcification is an independent stroke predictor in the general population. Stroke. 2013;44(4):1008-1013.

47. O'Neal WT, Efird JT, Dawood FZ, et al. Coronary artery calcium and risk of atrial fibrillation (from the multi-ethnic study of atherosclerosis). Am J Cardiol. 2014;114(11):1707-1712.

48. O'Neal WT, Efird JT, Qureshi WT, et al. Coronary artery calcium progression and atrial fibrillation: the multi-ethnic study of atherosclerosis. Circ Cardiovasc Imaging. 2015;8(12):e003786.

49. Pan NH, Tsao HM, Chang NC, Lee CM, Chen YJ, Chen SA. Dilated left atrium and pulmonary veins in patients with calcified coronary artery: a potential contributor to the genesis of atrial fibrillation. J Cardiovasc Electrophysiol. 2009;20(2):153-158.

50. Elmariah S, Budoff MJ, Delaney JA, et al. Risk factors associated with the incidence and progression of mitral annulus calcification: the multiethnic study of atherosclerosis. Am Heart J. 2013;166(5):904-912.

51. Kanjanauthai S, Nasir K, Katz R, et al. Relationships of mitral annular calcification to cardiovascular risk factors: the Multi-Ethnic Study of Atherosclerosis (MESA). Atherosclerosis. 2010;213(2):558-562.

52. Kizer JR, Bella JN, Palmieri V, et al. Left atrial diameter as an independent predictor of first clinical cardiovascular events in middleaged and elderly adults: the Strong Heart Study (SHS). Am Heart J. 2006;151(2):412-418.

53. Fox CS, Parise H, Vasan RS, et al. Mitral annular calcification is a predictor for incident atrial fibrillation. Atherosclerosis. 2004;173(2):291-294.

54. O'Neal WT, Efird JT, Nazarian S, Alonso A, Heckbert SR, Soliman EZ. Mitral annular calcification and incident atrial fibrillation in the MultiEthnic study of Atherosclerosis. Europace. 2015;17(3):358-363.

55. Selvin E, Erlinger TP. Prevalence of and risk factors for peripheral arterial disease in the United States: results from the National Health and Nutrition Examination Survey, 1999-2000. Circulation. 2004;110(6):738-743.

56. Anderson JL, Halperin JL, Albert NM, et al. Management of patients with peripheral artery disease (compilation of 2005 and $2011 \mathrm{ACCF} /$ AHA guideline recommendations): a report of the American College of Cardiology Foundation/American Heart Association Task Force on Practice Guidelines. Circulation. 2013;127(13):1425-1443.

57. Perlstein TS, Creager MA. The ankle-brachial index as a biomarker of cardiovascular risk: it's not just about the legs. Circulation. 2009;120(21):2033-2035.

58. O’Neal WT, Efird JT, Nazarian S, Alonso A, Heckbert SR, Soliman EZ. Peripheral arterial disease and risk of atrial fibrillation and stroke: the Multi-Ethnic study of atherosclerosis. J Am Heart Assoc. 2014;3(6): e001270.

59. Griffin WF, Salahuddin T, O’Neal WT, Soliman EZ. Peripheral arterial disease is associated with an increased risk of atrial fibrillation in the elderly. Europace. 2016;18(6):794-8.

60. Hancock EW, Deal BJ, Mirvis DM, et al. AHA/ACCF/HRS recommendations for the standardization and interpretation of the electrocardiogram: part V: electrocardiogram changes associated with cardiac chamber hypertrophy: a scientific statement from the American Heart Association Electrocardiography and Arrhythmias Committee, Council on Clinical Cardiology; the American College of Cardiology Foundation; and the Heart Rhythm Society. Endorsed by the International Society for Computerized Electrocardiology. $\mathrm{J} \mathrm{Am} \mathrm{Coll} \mathrm{Cardiol.}$ 2009;53(11):992-1002. 
61. Iwasaki YK, Nishida K, Kato T, Nattel S. Atrial fibrillation pathophysiology: implications for management. Circulation. 2011;124(20): 2264-2274.

62. Heikkila J, Hugenholtz PG, Tabakin BS. Prediction of left heart filling pressure and its sequential change in acute myocardial infarction from the terminal force of the P wave. Br Heart J. 1973;35(2):142-151.

63. Kasser I, Kennedy JW. The relationship of increased left atrial volume and pressure to abnormal $\mathrm{P}$ waves on the electrocardiogram. Circulation. 1969;39(3):339-343.

64. Soliman EZ, Prineas RJ, Case LD, Zhang ZM, Goff DC Jr. Ethnic distribution of ECG predictors of atrial fibrillation and its impact on understanding the ethnic distribution of ischemic stroke in the Atherosclerosis Risk in Communities (ARIC) study. Stroke. 2009;40(4):1204-1211.

65. Magnani JW, Johnson VM, Sullivan LM, et al. P wave duration and risk of longitudinal atrial fibrillation in persons $\geq 60$ years old (from the Framingham Heart Study). Am J Cardiol. 2011;107(6):917-921, e911.

66. Nielsen JB, Kuhl JT, Pietersen A, et al. P-wave duration and the risk of atrial fibrillation: results from the Copenhagen ECG study. Heart Rhythm. 2015;12(9):1887-1895.

67. Cheng S, Keyes MJ, Larson MG, et al. Long-term outcomes in individuals with prolonged PR interval or first-degree atrioventricular block. JAMA. 2009;301(24):2571-2577.

68. Magnani JW, Wang N, Nelson KP, et al. Electrocardiographic PR interval and adverse outcomes in older adults: the Health, Aging, and Body Composition study. Circ Arrhythm Electrophysiol. 2013;6(1):84-90.

69. Nielsen JB, Pietersen A, Graff C, et al. Risk of atrial fibrillation as a function of the electrocardiographic PR interval: results from the Copenhagen ECG Study. Heart Rhythm. 2013;10(9):1249-1256.
70. Alonso A, Krijthe BP, Aspelund T, et al. Simple risk model predicts incidence of atrial fibrillation in a racially and geographically diverse population: the CHARGE-AF consortium. J Am Heart Assoc. 2013;2(2):e000102.

71. Schnabel RB, Sullivan LM, Levy D, et al. Development of a risk score for atrial fibrillation (Framingham Heart Study): a community-based cohort study. Lancet. 2009;373(9665):739-745.

72. Chamberlain AM, Agarwal SK, Folsom AR, et al. A clinical risk score for atrial fibrillation in a biracial prospective cohort (from the Atherosclerosis Risk in Communities [ARIC] study). Am J Cardiol. 2011;107(1): 85-91.

73. Burstein B, Nattel S. Atrial fibrosis: mechanisms and clinical relevance in atrial fibrillation. J Am Coll Cardiol. 2008;51(8):802-809.

74. Casaclang-Verzosa G, Gersh BJ, Tsang TS. Structural and functional remodeling of the left atrium: clinical and therapeutic implications for atrial fibrillation. J Am Coll Cardiol. 2008;51(1):1-11.

75. O’Neal WT, Soliman EZ, Qureshi W, Alonso A, Heckbert SR, Herrington D. Sustained pre-hypertensive blood pressure and incident atrial fibrillation: the Multi-Ethnic Study of Atherosclerosis. J Am Soc Hypertens. 2015;9(3):191-196.

76. Healey JS, Baranchuk A, Crystal E, et al. Prevention of atrial fibrillation with angiotensin-converting enzyme inhibitors and angiotensin receptor blockers: a meta-analysis. J Am Coll Cardiol. 2005;45(11): 1832-1839.

77. Burgstahler C, Reimann A, Beck T, et al. Influence of a lipid-lowering therapy on calcified and noncalcified coronary plaques monitored by multislice detector computed tomography: results of the New Age II Pilot Study. Invest Radiol. 2007;42(3):189-195.
Vascular Health and Risk Management

\section{Publish your work in this journal}

Vascular Health and Risk Management is an international, peerreviewed journal of therapeutics and risk management, focusing on concise rapid reporting of clinical studies on the processes involved in the maintenance of vascular health; the monitoring, prevention and treatment of vascular disease and its sequelae; and the involvement of

\section{Dovepress}

metabolic disorders, particularly diabetes. This journal is indexed on PubMed Central and MedLine. The manuscript management system is completely online and includes a very quick and fair peer-review system, which is all easy to use. Visit http://www.dovepress.com/ testimonials.php to read real quotes from published authors. 